TRANSACTIONS OF THE

AMERICAN MATHEMATICAL SOCIETY

Volume 359, Number 6, June 2007, Pages 2663-2673

S 0002-9947(07)04127-X

Article electronically published on January 19, 2007

\title{
CLOSED TIMELIKE GEODESICS IN COMPACT SPACETIMES
}

\author{
MOHAMMED GUEDIRI
}

\begin{abstract}
Let $M$ be a compact spacetime which admits a regular globally hyperbolic covering, and $\mathcal{C}$ a nontrivial free timelike homotopy class of closed timelike curves in $M$. We prove that $\mathcal{C}$ contains a longest curve (which must be a closed timelike geodesic) if and only if the timelike injectivity radius of $\mathcal{C}$ is finite; i.e., $\mathcal{C}$ has a bounded length. As a consequence among others, we deduce that for a compact static spacetime there exists a closed timelike geodesic within every nontrivial free timelike homotopy class having a finite timelike injectivity radius.
\end{abstract}

\section{INTRODUCTION}

An old existence theorem (cf. [5], [14, or [18]) for closed geodesics on Riemannian manifolds asserts that in any nontrivial free homotopy class of closed curves on a compact Riemannian manifold there exists a shortest curve, which must be a nontrivial closed geodesic.

Very little is known at present concerning an analogue of this result for compact spacetimes. In a spacetime, there are three types of geodesics: timelike, null (lightlike), and spacelike geodesics. Timelike and null geodesics represent, respectively, free falling particles and light rays, while a spacelike geodesic has no physical meaning given that it should represent a particle moving faster than light. Accordingly, it would be of interest to obtain a corresponding result for nonspacelike geodesics. Actually, since timelike geodesics maximize Lorentz distance between two points and since any curve can be deformed into a null curve which has zero length, we seek the longest curve instead of the shortest curve. Given a nontrivial free timelike homotopy class $\mathcal{C}$ of closed timelike curves on a compact spacetime $(M, g)$, it is evident that a necessary condition for the existence of a longest curve in $\mathcal{C}$ (which must be a closed timelike geodesic) is that $\mathcal{C}$ should be bounded in length (we shall say in such a case that $\mathcal{C}$ has a finite timelike injectivity radius). Accordingly, it is natural to ask whether this necessary condition is also a sufficient condition for the existence of a longest curve in a given $\mathcal{C}$.

As Galloway noticed in [6], p. 384, one can conceive the difficulty that a sequence of curves in $\mathcal{C}$ whose lengths approach the supremum of lengths of all curves that belong to $\mathcal{C}$ may be approaching a closed null geodesic, and to overcome this difficulty he made a stronger assumption (called of stability) that $\mathcal{C}$ is bounded in length with respect to a new Lorentz metric wider than $g$. He established the existence of a longest curve (i.e., a closed timelike geodesic) in every stable nontrivial

Received by the editors March 16, 2005.

2000 Mathematics Subject Classification. Primary 53C50, 53C22.

(C) 2007 American Mathematical Society 
free timelike homotopy class of closed timelike curves on a compact spacetime. Previously, Tipler [19] established the existence of a closed timelike geodesic in every compact spacetime admitting a regular covering that contains a compact Cauchy hypersurface.

Very recently, we provided in [9] and [11 the first examples of compact flat spacetimes with no closed timelike geodesics. Since the universal covering of such spacetimes is a Minkowski spacetime, this obviously shows that when the Cauchy hypersurfaces are not required to be compact then Tipler's result does not hold if no additional condition is made. On the other hand, we proved in [9] and [10] that on a compact spacetime with a regular globally hyperbolic covering (i.e., the Cauchy hypersurfaces are not necessarily compact) there always exists a longest curve (which is a closed timelike geodesic) within every nontrivial free timelike homotopy class which is determined by a deck transformation which is central or a Clifford translation. As an immediate consequence of this, we derived that Tipler's result remains valid when the compactness assumption on the Cauchy hypersurfaces is replaced by the assumption that the fundamental group of the spacetime is abelian or consists purely of Clifford translations.

In connection with this, we should also mention the work of Wegner 20. By using the notion of quasi-limits first introduced in [15, pp. 404-407, he proved the following result. Let $M$ be a compact spacetime which is timelike geodesically complete, and $\mathcal{C}$ a nontrivial free timelike homotopy class which is bounded in length, and let $L$ denote the supremum of lengths of all curves that belong to $\mathcal{C}$. Then either $\mathcal{C}$ contains a closed timelike geodesic of length $L$, or for each sequence of curves in $\mathcal{C}$ whose lengths approach $L$ every quasi-limit curve is an inextendible null geodesic (which is not necessarily closed).

In this paper, we shall give an affirmative answer to the question we raised above in the important case of a compact spacetime admitting a regular globally hyperbolic covering space. The outline of the paper is as follows. In Section 2 we give some preliminary definitions and properties from causal theory. In particular, after presenting what we have introduced in 9 and called the timelike injectivity radius for a free t-homotopy class (recall that it was defined to be one-half the supremum of lengths of all closed curves belonging to that class) we show that, for a compact spacetime admitting a regular globally hyperbolic covering, the timelike injectivity radius is finite whenever the displacement function associated to the t-homotopy class is invariant under the action of the group of deck transformations. This is for instance the case when the deck transformation determining the t-homotopy class is central or a Clifford translation. Section 3 is the main part of this paper. It contains our main result which provides an affirmative answer to the question raised above in the important case of a compact spacetime admitting a globally hyperbolic covering space. This improves Tipler's result [19] mentioned above and the results in 9 and [10. In Section 4, by combining our main result with Synge's lemma for Lorentzian manifolds in [6, we derive a Lorentzian version of the well known Synge's theorem. In the last section (Section 5), we present some applications of the main theorem in the important case of a static spacetime; that is, a spacetime which admits a timelike Killing field whose orthogonal distribution is integrable. Namely, we prove that in a compact static spacetime, every free t-homotopy class with finite timelike injectivity radius contains a closed timelike geodesic. This in particular establishes the existence of closed timelike geodesics in every compact 
static spacetime with fundamental group that is either abelian, consists purely of Clifford translations, or has the property that every conjugacy class in it is finite.

\section{Preliminaries}

We recall the basic definitions from causal theory which will be needed in this paper. Complete details may be found in [1, 13, 15].

Throughout this paper, a spacetime $(M, g)$ will indicate a connected manifold $M$ of dimension $n \geq 2$ with a Lorentz metric $g$ of signature convention $(-,+, \ldots,+)$ such that $(M, g)$ is assumed to be time-orientable. A tangent vector $X$ to $M$ is said to be timelike, spacelike or null (lightlike) according to whether $g(X, X)<0,>0$ or $=0$, respectively. Similarly, a smooth curve in $M$ is said to be timelike, spacelike or null if its tangent vector field is always timelike, spacelike or null, respectively.

2.1. Globally hyperbolic spacetimes. A fundamental concept in Lorentz geometry is global hyperbolicity. It is defined as follows: Let $(M, g)$ be a spacetime. For a given $x \in M$, the set

$$
J^{+}(x)=\{y \in M: \text { there is a future directed nonspacelike curve from } x \text { to } y\}
$$

is called the causal future of $x$. The causal past $J^{-}(x)$ of $x$ is defined dually. By definition, $(M, g)$ is said to be globally hyperbolic if it is strongly causal and the set $J^{+}(x) \cap J^{-}(y)$ is compact for all $x, y \in M$. Recall that $(M, g)$ is strongly causal if for each $x \in M$, nonspacelike curves that start arbitrarily close to $x$ and leave some fixed neighborhood cannot return arbitrarily close to $x$. The idea here is to say that there is no almost closed nonspacelike curves.

Global hyperbolicity can also be characterized in terms of Cauchy hypersurfaces. A subset $S$ of $M$ is a Cauchy hypersurface if and only if each inextendible nonspacelike curve intersects $S$ once and only once. A basic result (see [13]) of causal theory asserts that a spacetime is globally hyperbolic if and only if it admits a Cauchy hypersurface.

2.2. The Lorentzian distance. The Lorentzian distance function $d_{g}: M \times M \rightarrow$ $\overline{\mathbb{R}}_{+}$for a spacetime $(M, g)$ is defined as follows. Given $x, y \in M$, let $C(x, y)$ denote the set of all future-directed nonspacelike curves from $x$ to $y$ which are piecewise smooth. Set $d_{g}(x, y)=0$ if $y \notin J^{+}(x)$ and $d_{g}(x, y)=\sup \left\{L_{g}(\gamma): \gamma \in C(x, y)\right\}$ if $y \in J^{+}(x)$, where $L_{g}(\gamma)$ is the length of $\gamma$ relative to $g$. Unlike the Riemannian distance, the Lorentzian distance function $d_{g}$ is not in general upper semicontinuous and it may be infinite. The main case of interest to us in this paper is when $(M, g)$ (or a covering of it) is assumed globally hyperbolic. In this case, $d_{g}$ is finite and continuous on the whole $M \times M$ (see [13]).

2.3. Globally hyperbolic covering spaces. A covering $\pi: \widetilde{M} \rightarrow M$ is said to be regular if for any $x, y \in \widetilde{M}$ such that $\pi(x)=\pi(y)$, there exists a deck transformation $\phi$ such that $\phi(x)=y$. This means that the group of deck transformations $\Gamma$ of $\pi$ acts transitively on each fibre $\pi^{-1}(x)$. For instance, every simply connected covering space is regular and we have in that case $\Gamma \cong \pi_{1}(M)$ (cf. [21]).

Now suppose that $\pi: \widetilde{M} \rightarrow M$ is a differentiable covering with $M$ endowed with a Lorentzian metric $g$, and endow $\widetilde{M}$ with the Lorentzian metric $\widetilde{g}$ for which $\pi$ becomes a Lorentzian covering (that is, $\pi$ is a local isometry). It follows that $\Gamma$ already acting freely and properly discontinuously further acts isometrically on 
$\widetilde{M}$. If in addition $\pi$ is regular and $(M, g)$ is geodesically complete, then we say that the deck transformation $\phi$ translates the geodesic $\widetilde{\gamma}(t)$ of $\widetilde{M}$ an amount $\omega$ if $\phi . \widetilde{\gamma}(t)=\widetilde{\gamma}(t+\omega)$ for all $t \in \mathbb{R}$. In this case, it is clear that $\widetilde{\gamma}$ projects via $\pi$ onto a closed geodesic $\pi \circ \widetilde{\gamma}$ in $M$ and conversely every closed geodesic in $M$ is obtained in this manner. In the case $\widetilde{M}$ is globally hyperbolic and $\widetilde{\gamma}(t)$ is a unit speed geodesic, the positive number $\omega$ (called a period of $\phi$ ) coincides with the length of the closed (non-null) geodesic $\pi \circ \widetilde{\gamma}$.

2.4. The timelike homotopy. Let $(M, g)$ be a spacetime and $\pi: \widetilde{M} \rightarrow M$ a regular globally hyperbolic covering. Two closed timelike curves $\gamma_{1}$ and $\gamma_{2}$ in $M$ are said to be freely timelike homotopic (or briefly: freely t-homotopic) if there is a homotopy which deforms $\gamma_{1}$ to $\gamma_{2}$ via closed timelike curves. If $\mathcal{C}$ is a t-homotopy class of closed timelike curves in $M$, let $\gamma:[0,1] \rightarrow M$ be a closed timelike curve belonging to $\mathcal{C}$. Let $\widetilde{\gamma}:[0,1] \rightarrow \widetilde{M}$ be a lift of $\gamma$ starting at some $\widetilde{p}_{1} \in \pi^{-1}(p)$, where $p=\gamma(0)=\gamma(1)$, and set $\widetilde{p}_{2}=\widetilde{\gamma}(1)$. Since $\widetilde{M}$ is globally hyperbolic, we have $\widetilde{p}_{1} \neq \widetilde{p}_{2}$. Without loss of generality, we may assume that there exists a future directed timelike curve from $\widetilde{p}_{1}$ to $\widetilde{p}_{2}$, and since $\pi$ is regular there is a unique $\phi \in \Gamma$ such that $\widetilde{p}_{2}=\phi\left(\widetilde{p}_{1}\right)$. Moreover, since any other point $\widetilde{q} \in \pi^{-1}(p)$ is of the form $\widetilde{q}=\psi\left(\widetilde{p}_{1}\right)$ for some $\psi \in \Gamma$, we see that the lift of $\gamma$ starting at $\widetilde{q}$ is $\psi \circ \widetilde{\gamma}$. It follows that

$$
\begin{aligned}
\psi \circ \widetilde{\gamma}(1) & =\psi\left(\widetilde{p}_{2}\right) \\
& =\psi \circ \phi\left(\widetilde{p}_{1}\right) \\
& =\psi \circ \phi(\widetilde{\gamma}(0)) \\
& =\psi \circ \phi \circ \psi^{-1}(\psi \circ \widetilde{\gamma}(0)) .
\end{aligned}
$$

Thus, the conjugacy class $[\phi]=\left\{\psi \circ \phi \circ \psi^{-1}: \psi \in \Gamma\right\}$ does not depend on the choice of the point $\widetilde{p} \in \pi^{-1}(p)$ at which we lift $\gamma$. Furthermore, one may check straightforwardly that any other closed timelike curve in $M$ which is freely thomotopic to $\gamma$ determines the same conjugacy class $[\phi]$ in $\Gamma$. Hence, $[\phi]$ is nothing but the conjugacy class in $\Gamma$ representing $\mathcal{C}$. We deduce that there is a one-to-one (but not onto) correspondence between the set of free t-homotopy classes of closed timelike curves in $M$ and the conjugacy classes in the group $\Gamma$ of deck transformations of the covering $\pi: \widetilde{M} \rightarrow M$.

2.5. The timelike injectivity radius of a t-homotopy class. In [9, we introduced for a free t-homotopy class the notion of timelike injectivity radius, as follows. Given a free t-homotopy class $\mathcal{C}$ of closed timelike curves in a spacetime $(M, g)$, we define the timelike injectivity radius of $\mathcal{C}$ to be

$$
\operatorname{Tinj}(\mathcal{C})=\frac{1}{2} \sup _{\gamma \in \mathcal{C}} L_{g}(\gamma)
$$

where $L_{g}(\gamma)$ is the length of $\gamma$ with respect to the metric $g$.

Now let $(M, g)$ be a spacetime with a regular Lorentzian covering space $(\widetilde{M}, \widetilde{g})$ that we assume globally hyperbolic. Let $\pi: \widetilde{M} \rightarrow M$ be the covering map, and $\widetilde{d}$ the Lorentzian distance on $\widetilde{M}$ associated to $\widetilde{g}$ (obviously, we have $\widetilde{g}=\pi^{*} g$ ). Let $\mathcal{C}$ be a non-trivial free t-homotopy class in $M$ that is determined by a non-trivial deck transformation $\phi$. By using the notation of the subsections above, one can define the mapping (called the displacement function) $f_{\phi}: \widetilde{M} \rightarrow \mathbb{R}$ by $f_{\phi}(x)=\widetilde{d}(x, \phi(x)$ ), 
which must be continuous as $\widetilde{M}$ is globally hyperbolic. Define the mapping $h_{\phi}$ : $\widetilde{M} \rightarrow \mathbb{R}$ by $h_{\phi}(x)=\sup \left\{f_{\phi}(\psi(x)): \psi \in \Gamma\right\}$.

Since $f_{\phi}(\psi(x))=\widetilde{d}\left(x, \psi^{-1} \circ \phi \circ \psi(x)\right)$, we deduce that

$$
\operatorname{Tinj}(\mathcal{C})=\frac{1}{2} \sup _{\gamma \in \mathcal{C}} L_{g}(\gamma)=\frac{1}{2} \sup _{x \in \widetilde{M}} h_{\phi}(x) .
$$

Proposition 2.1. Let $(M, g)$ be a compact spacetime with a regular globally hyperbolic covering $\pi: \widetilde{M} \rightarrow M$, and $\mathcal{C}$ a non-trivial free t-homotopy class of closed timelike curves in $M$ that is determined by a deck transformation $\phi$ for which the displacement function $f_{\phi}$ is invariant under the action of the group $\Gamma$ of deck transformations of $\pi$. Then Tinj $(\mathcal{C})<\infty$.

Proof. Let $\mathcal{C}$ be a non-trivial free t-homotopy class of closed curves in $M$ that is determined by a non-trivial deck transformation $\phi \in \Gamma$, for which the displacement function $f_{\phi}: \widetilde{M} \rightarrow \mathbb{R}$ associated to $\phi$ is $\Gamma$-invariant. Since the fundamental domains for the action of $\Gamma$ are compact, it follows that $f_{\phi}$ attains its maximum value on each compact domain $K \subset \widetilde{M}$. But $\Gamma(K)=\widetilde{M}$ and $f_{\phi}$ is assumed $\Gamma$-invariant, hence the maximum of $f_{\phi}$ on $K$ is also the maximum on all of $\widetilde{M}$. By (2.1) and the invariance of $f_{\phi}$ we conclude that

$$
\operatorname{Tinj}(\mathcal{C})=\frac{1}{2} \sup _{x \in \widetilde{M}} h_{\phi}(x)=\frac{1}{2} \sup _{x \in \widetilde{M}} f_{\phi}(x)=\frac{1}{2} \sup _{x \in K} f_{\phi}(x)<\infty,
$$

as required.

As an immediate consequence of Proposition 2.1. we obtain the following result which is contained in Theorem 3.1 of [10]. Before stating it, recall that an isometry $\phi$ of a spacetime $(M, g)$ is called a Clifford translation if $d_{g}(x, \phi(x))=$ const. for all $x \in M$.

Corollary 2.2. Let $(M, g)$ be a compact spacetime with a regular globally hyperbolic covering $\pi: \widetilde{M} \rightarrow M$, and $\mathcal{C}$ a non-trivial free t-homotopy class of closed timelike curves in $M$ that is determined by a deck transformation $\phi$ which is central or a Clifford translation. Then $\operatorname{Tinj}(\mathcal{C})<\infty$.

Remark 1. It may be worth noting that $\operatorname{Tinj}(\mathcal{C})$ need not be finite even if $M$ is compact (see 6], p. 380, for an explicit example).

\section{The MAIN RESUlT}

As mentioned in the Introduction, a necessary condition for the existence of a closed timelike geodesic in a given non-trivial free t-homotopy class $\mathcal{C}$ of closed timelike curves in a compact spacetime $(M, g)$ is

$$
\operatorname{Tinj}(\mathcal{C})<\infty .
$$

Question. Is this condition sufficient?

We do not know whether the answer to this question is yes or no in general, but as Galloway mentioned in [6], p. 384, even if $M$ is geodesically complete one can conceive of the following difficulty: If $\mathcal{C}$ is a non-trivial free t-homotopy class satisfying (3.1), then a sequence of curves in $\mathcal{C}$ whose lengths approach $\operatorname{Tinj}(\mathcal{C})$ may be approaching a closed null geodesic. However, if we restrict our attention 
to the class of compact spacetimes admitting a globally hyperbolic covering, then things work nicely and the answer to the above question is affirmative, namely we obtain the following theorem which is the main result of this article. Its proof is inspired from the first proof of Proposition 25, p. 355, of [18].

Theorem 3.1. Let $(M, g)$ be a compact spacetime with a regular globally hyperbolic covering $\pi: \widetilde{M} \rightarrow M$, and $\mathcal{C}$ a non-trivial free t-homotopy class of closed timelike curves in $M$. Then $\mathcal{C}$ contains a longest curve which must be a non-trivial closed timelike geodesic if and only if $\operatorname{Tinj}(\mathcal{C})<\infty$.

Proof. In one direction, this is obvious as mentioned above. In the other direction, let us assume that $\mathcal{C}$ is a non-trivial free t-homotopy class such that $\operatorname{Tinj}(\mathcal{C})<\infty$, and let $\phi$ be a deck transformation representing the conjugacy class corresponding to $\mathcal{C}$. Since $\widetilde{M}$ is globally hyperbolic, we deduce from (2.1) that, for all $x \in \widetilde{M}$, we have

$$
h_{\phi}(x)=\sup \left\{\widetilde{d}\left(x, \psi^{-1} \circ \phi \circ \psi(x)\right): \psi \in \Gamma\right\}<\infty .
$$

Since the fibres $\pi^{-1}(y)$ are discrete, it is immediate from (3.2) that for each $x \in \widetilde{M}$ there exists a deck transformation $\widetilde{\psi}$ which depends on $x$ such that $h_{\phi}(x)=$ $\widetilde{d}\left(x, \widetilde{\psi}^{-1} \circ \phi \circ \widetilde{\psi}(x)\right)$. On the other hand, as in the proof of Proposition 2.1, since the fundamental domains for the action of $\Gamma$ are compact and $\Gamma(K)=\widetilde{M}$ for each compact domain $K \subset \widetilde{M}$, it follows by the $\Gamma$-invariance of $h_{\phi}$ (which is obvious) that $h_{\phi}$ achieves its maximum value at some $x_{0} \in \widetilde{M}$; that is, $h_{\phi}\left(x_{0}\right)=$ $\sup \left\{h_{\phi}(x): x \in \widetilde{M}\right\}$. Note that $h_{\phi}\left(x_{0}\right)>0$, given that $\mathcal{C}$ is assumed to be nontrivial. Let $\widetilde{\psi}_{0} \in \Gamma$ such that $h_{\phi}\left(x_{0}\right)=\widetilde{d}\left(x_{0}, \widetilde{\psi}_{0}^{-1} \circ \phi \circ \widetilde{\psi}_{0}\left(x_{0}\right)\right)$ and let $\widetilde{\gamma}:[0,1] \rightarrow$ $\widetilde{M}$ be a timelike geodesic from $x_{0}$ to $x_{1}=\widetilde{\psi}_{0}^{-1} \circ \phi \circ \widetilde{\psi}_{0}\left(x_{0}\right)$ realizing this maximum value of length; that is, $h_{\phi}\left(x_{0}\right)=L_{\widetilde{g}}(\widetilde{\gamma})$.

By projecting $\widetilde{\gamma}$ under $\pi$ we obtain a closed timelike geodesic $\sigma=\pi \circ \widetilde{\gamma}$ in $M$ which must belong to $\mathcal{C}$. It remains to prove that $\sigma$ is the longest curve in $\mathcal{C}$. To this end, let $c:[0,1] \rightarrow M$ be any other curve in $\mathcal{C}$, and let $\widetilde{c}:[0,1] \rightarrow \widetilde{M}$ be a lifting starting at some point $p=\widetilde{c}(0)$. In this case, there is some $\delta \in \Gamma$ such that $\widetilde{c}(1)=\delta^{-1} \circ \phi \circ \delta(p)$. It follows that

$$
\begin{aligned}
L_{g}(c) & =L_{\widetilde{g}}(\widetilde{c}) \\
& \leq \widetilde{d}\left(p, \delta^{-1} \circ \phi \circ \delta(p)\right) \\
& \leq h_{\phi}(p) \\
& \leq h_{\phi}\left(x_{0}\right) \\
& =L_{\widetilde{g}}(\widetilde{\gamma})=L_{g}(\sigma) .
\end{aligned}
$$

This proves that $\sigma$ is the longest curve in $\mathcal{C}$. In particular, we deduce that $\sigma$ must be an unbroken closed geodesic (see [13], pp. 106-107). The proof of the main result is complete.

Remark 2. As Galloway communicated to me, Theorem 3.1 may be viewed as the natural Lorentzian analogue of Proposition 25 of [18], and then it seems to be the most natural statement for compact spacetimes admitting regular globally hyperbolic coverings. The analogy goes a bit further due to the fact that any covering 
of a globally hyperbolic spacetime is globally hyperbolic. Thus the assumption of a regular globally hyperbolic covering is equivalent to the assumption that the universal cover is globally hyperbolic.

As an immediate consequence of Theorem 3.1 and Corollary 2.2, we have the following result which is Theorem 3.1 of [10].

Proposition 3.2. Let $(M, g)$ be a compact spacetime with a regular globally hyperbolic covering $\pi: \widetilde{M} \rightarrow M$, and $\mathcal{C}$ a non-trivial free t-homotopy class of closed timelike curves in $M$ that is determined by a deck transformation $\phi$ which is either central, a Clifford translation, or else the conjugacy class of $\phi$ is finite. Then $\mathcal{C}$ contains a longest curve which must be a non-trivial closed timelike geodesic.

Since any compact spacetime contains a nontrivial timelike curve, then by considering the conjugacy class representing the free t-homotopy class to which this curve belongs, we deduce from the above proposition the following result which is Theorem 3.2 of [10].

Proposition 3.3. Let $(M, g)$ be a compact spacetime with a regular globally hyperbolic covering $\pi: \widetilde{M} \rightarrow M$. If the group of deck transformations $\Gamma$ of $\pi$ is abelian, consists purely of Clifford translations, or every conjugacy class in $\Gamma$ is finite, then $(M, g)$ contains a closed timelike geodesic.

Another consequence of Theorem 3.1 is the following.

Proposition 3.4. Let $N$ be a simply connected 2-step nilpotent Lie group with a flat left invariant Lorentz metric, and $\Lambda$ a lattice in $N$. If $\mathcal{C}$ is a non-trivial free t-homotopy class in the compact, flat, Lorentz 2-step nilmanifold $N / \Lambda$, then $\operatorname{Tinj}(\mathcal{C})=\infty$.

Proof. This follows from Theorem 3.1] and Theorem 3 of [11] which states that if $N$ is a simply connected 2-step nilpotent Lie group with a flat left invariant Lorentz metric $g$, and $\Lambda$ a discrete subgroup of $N$ (not necessarily cocompact), then the flat Lorentz 2-step nilmanifold $N / \Lambda$ contains no closed timelike geodesics.

\section{Around a Lorentzian version of Synge's theorem}

The well known Synge's theorem on connectivity (cf. [18, p. 353) states that a compact, connected, orientable, even-dimensional Riemannian manifold with all sectional curvatures positive is simply connected. In [6], Galloway presented a Lorentzian analogue of this result by proving that, for a spacetime $(M, g)$, the following assertions are incompatible:

1. $M$ is compact, even-dimensional, and orientable.

2. Every timelike plane section $\mathcal{P}$ in $M$ has sectional curvature $K(\mathcal{P})<0$.

3. $M$ has a stable t-homotopy class; that is, there exist a free t-homotopy class $\mathcal{C}$ of closed timelike curves in $(M, g)$ and a Lorentzian metric $g_{1}$ wider than $g$ for which $\operatorname{Tinj}(\mathcal{C})<\infty$.

We present here another Lorentzian version of Synge's theorem.

Theorem 4.1. For a spacetime $(M, g)$, the following assertions are incompatible:

1. $M$ is compact, even-dimensional, and orientable.

2. Every timelike plane section $\mathcal{P}$ in $M$ has sectional curvature $K(\mathcal{P})<0$. 
3. $M$ admits a regular globally hyperbolic covering, and there is a free $t$ homotopy class $\mathcal{C}$ in $M$ such that $\operatorname{Tinj}(\mathcal{C})<\infty$.

Proof. This follows by combining Theorem 3.1 with Synge's lemma for Lorentzian manifolds in [6] which states that if $M$ is an even-dimensional, orientable spacetime with $K(\mathcal{P})<0$ for all timelike plane sections $\mathcal{P}$, then for any closed timelike geodesic $\gamma$ in $M$ there exists a variation of $\gamma$ by timelike curves such that each varied curve has length greater than $\gamma$.

More particularly, we obtain the following.

Proposition 4.2. Let $(M, g)$ be a compact, connected, orientable, and even-dimensional spacetime with a globally hyperbolic covering and such that every timelike plane section $\mathcal{P}$ in $M$ has sectional curvature $K(\mathcal{P})<0$. Then Tinj $(\mathcal{C})=\infty$ for every free t-homotopy class $\mathcal{C}$ in $M$.

From the latter, by considering the case $M=T^{2 n}$ a torus of dimension $2 n$, we obtain the following result.

Proposition 4.3. Let $T^{2 n}$ be a $2 n$-dimensional Lorentz torus with a regular globally hyperbolic covering. Then, there exists a timelike plane section $\mathcal{P}$ with sectional curvature $K(\mathcal{P}) \geq 0$.

Proof. Assume to the contrary that every timelike plane section $\mathcal{P}$ in $T^{2 n}$ has sectional curvature $K(\mathcal{P})<0$. In this case, by Proposition 4.2, we have $\operatorname{Tinj}(\mathcal{C})=$ $\infty$ for every free t-homotopy class $\mathcal{C}$. But this contradicts Corollary 2.2, given that $T^{2 n}$ (as a compact spacetime) contains a non-trivial timelike curve which in turn determines a non-trivial t-homotopy class. This contradiction shows that necessarily $K(\mathcal{P}) \geq 0$ for some timelike plane section $\mathcal{P}$.

Remark 3. It follows from Corollary 2.2 that Proposition 4.3 remains valid if $T^{2 n}$ is replaced by any compact even-dimensional manifold having a fundamental group which is abelian or consists purely of Clifford translations.

\section{Closed geodesics in COMPaCt Static spacetimes}

It is well known (see [8]) that a compact spacetime admitting a timelike Killing vector field is geodesically complete. A natural question then arises: Does such a spacetime contain a closed timelike geodesic? We do not have an immediate answer to this question in the general case, but we know at least that the answer is yes in the two-dimensional case.

Proposition 5.1. Every compact two-dimensional spacetime admitting a timelike (or spacelike) Killing vector field contains a closed timelike geodesic.

Proof. Let $\left(M^{2}, g\right)$ be a compact two-dimensional spacetime admitting a timelike (or spacelike) Killing vector field $X$. Note first that, up to an appropriate finite covering, one can assume that $M^{2}$ is the Lorentz torus $T^{2}$. By Theorem 2.3 of [17, we get that $T^{2}$ is globally conformally flat. This implies that the universal covering $\widetilde{T^{2}}$ of $T^{2}$ is globally conformal to Minkowski space, and hence $\widetilde{T^{2}}$ is globally hyperbolic. Since the fundamental group of $M^{2}$ is abelian, it follows from Proposition 3.3 that $M^{2}$ contains a closed timelike geodesic. 
It is worth mentioning that the orthogonal distribution to a timelike (or spacelike) Killing vector field on a Lorentz 2-torus $T^{2}$ is always integrable, and that every free t-homotopy class $\mathcal{C}$ of closed timelike curves in $T^{2}$ satisfies (3.1) because $\pi_{1}\left(T^{2}\right)$ is abelian. Accordingly, it is natural to expect an affirmative answer to the question above in higher dimension under the two additional assumptions that the orthogonal distribution to the timelike Killing field is integrable and every free t-homotopy class $\mathcal{C}$ of closed timelike curves in $M$ satisfies (3.1).

Definition 5.2. A spacetime $M$ is said to be static if it admits a timelike vector field $X$ which is irrotational; that is, the curl of $X$ vanishes on the orthogonal distribution $X^{\perp}$.

Equivalently (see [15], p. 360), a spacetime $M$ is static if and only if there exists a timelike vector field $X$ whose orthogonal distribution $X^{\perp}$ is integrable and such that $h X$ is a Killing field for some positive smooth function $h$. See also 3 for a slightly different definition of a static spacetime.

The following result will be an essential tool for proving Theorem 5.5 below.

Theorem 5.3. Let $(M, g)$ be a compact spacetime which admits a spacelike codimension-one foliation $\mathcal{F}$ such that there exists a Killing vector field $X$ orthogonal to $\mathcal{F}$ everywhere, and let $\widetilde{M}$ be the universal covering of $M$ and $\pi: \widetilde{M} \rightarrow M$ the universal covering projection. Then $\widetilde{M}$ is isometric to a trivially foliated spacetime $\widetilde{L} \times \mathbb{R}$, where $\widetilde{L}$ is the universal covering of any leaf of $\mathcal{F}$ and the metric $\widetilde{g}=\pi^{*} g$ has the form $\widetilde{g}=d s_{\widetilde{L}}^{2}-f^{2} d t^{2}$, where $d s_{\widetilde{L}}^{2}$ is the Riemannian metric on $\widetilde{L}$ induced by $\widetilde{g}$ and $f$ is a smooth positive function on $\widetilde{M}$ invariant under the action of the fundamental group $\pi_{1}(M)$. In particular, $(\widetilde{M}, \widetilde{g})$ is globally hyperbolic.

Proof. By changing the sign of the metric $g$ on the orbits of $X$ we obtain a Riemannian metric $g_{1}$ on $M$ which must be complete, for $M$ is compact. With respect to $g_{1}$, we see that $X$ remains Killing and everywhere orthogonal to $\mathcal{F}$. Moreover, if $\widetilde{\mathcal{F}}$ denotes the canonical lifting of $\mathcal{F}$ to $\widetilde{M}$, we know from [16] that $\left(\widetilde{M}, \pi^{*} g\right)$, which is complete for the compactness of $M$, is trivially foliated as $\widetilde{M}=\widetilde{L} \times \mathbb{R}$, where $\widetilde{L}$ is the universal covering of any leaf of $\mathcal{F}$ and the metric $\widetilde{g}=\pi^{*} g$ on $\widetilde{M}$ is $\widetilde{g}=d s_{\widetilde{L}}^{2}+f^{2} d t^{2}$, where $f$ is a smooth positive function on $\widetilde{M}$ which must be invariant under the action of the group $\Gamma$ of deck transformations of $\widetilde{M}$, given that $M=\widetilde{M} / \Gamma$ (recall that $\Gamma$ is isomorphic to $\pi_{1}(M)$, as $\widetilde{M}$ is simply connected). The first conclusion of Theorem 5.3 now follows straightforwardly by again changing the sign of $\widetilde{g}$ on the orbits of the Killing field $\widetilde{X}$ on $\widetilde{M}, \pi$-related to $X$. To prove the second conclusion that $(\widetilde{M}, \widetilde{g})$ is globally hyperbolic, we first note that $(\widetilde{M}, \widetilde{g})$ must be geodesically complete, given that any compact spacetime which admits a timelike Killing vector field is geodesically complete (see [8]). On the other hand, it is well known in Riemannian geometry (see for instance [4) that a codimension-one foliation which admits an orthogonal Killing field is totally geodesic. This result can be generalized for Lorentzian manifolds. Accordingly, $\left(\widetilde{L}, d s_{\widetilde{L}}^{2}\right)$ is geodesically complete. Furthermore, since $f$ is $\Gamma$-invariant and the fundamental domains for the action of $\Gamma$ are compact, it follows that $f$ is bounded from below and from above. Thus, assumptions $\left(A_{1}\right)-\left(A_{3}\right)$ of Theorem 1.1 of [2] are satisfied, and hence $(\widetilde{M}, \widetilde{g})$ is globally hyperbolic (see Remark 1.3 in [2]). 
As a direct consequence of Theorem 5.3 is the following result.

Corollary 5.4. The universal covering $\widetilde{M}$ of a compact static spacetime $M$ is globally hyperbolic. More precisely, $\widetilde{M}$ is isometric to $\left(S \times \mathbb{R}, d s_{S}^{2}-f^{2} d t^{2}\right)$ where $S$ is a Cauchy hypersurface in $\widetilde{M}, d s_{S}^{2}$ is a Riemannian metric on $S$, and $f$ is a smooth positive function on $S \times \mathbb{R}$ which is invariant under the action of $\pi_{1}(M)$.

We can now state the main result of this section. This follows straightforwardly from Theorem 3.1 and Corollary 5.4

Theorem 5.5. Let $(M, g)$ be a compact static spacetime, and $\mathcal{C}$ a non-trivial free t-homotopy class in $M$ such that $\operatorname{Tinj}(\mathcal{C})<\infty$. Then $\mathcal{C}$ contains a longest curve which must be a non-trivial closed timelike geodesic.

As an immediate consequence of Theorem[5.5, we obtain the following interesting corollaries. The second one is Corollary 4.7 of $[3]$.

Corollary 5.6. Let $(M, g)$ be a compact static spacetime with $\pi_{1}(M)$ abelian, or consists purely of Clifford translations. Then $(M, g)$ contains a closed timelike geodesic.

Corollary 5.7. Let $(M, g)$ be a compact static spacetime, and assume that every conjugacy class in $\pi_{1}(M)$ is finite. Then $(M, g)$ contains a closed timelike geodesic.

\section{ACKNOWLEDGEMENTS}

The author wishes to thank Professor G. Galloway for the interest he has taken in this work.

\section{REFERENCES}

[1] J.K. Beem, P.E. Ehrlich, Global Lorentzian geometry, Pure and Applied Mathematics, Marcel Dekker Inc., 1996. MR:1384756 (97f:53100)

[2] V. Benci, D. Fortunato, A. Masiello, On the geodesic connectedness of Lorentzian manifolds, Math. Z. 217 (1994), 73-93. MR1292174 (96c:58037)

[3] E. Caponio, A. Masiello, P. Piccione, Some global properties of static spacetimes, Math. Z. 244 (2003), 457-468. MR.1992019 (2004c:53105)

[4] C. Currás-Bosch, Killing vector fields and holonomy algebras, Proc. Amer. Math. Soc. 90 (1984), 97-102. MR0722424 (85c:53068)

[5] S. Gallot, D. Hulin, J. Lafontaine, Riemannian geometry, Universitext, Berlin etc., SpringerVerlag, 1990. MR1083149 (91j:53001)

[6] G.J. Galloway, Closed timelike geodesics, Trans. Amer. Math. Soc. 285 (1984), 379-388. MR0748844 (85k:53061)

[7] G.J. Galloway, Compact Lorentz manifolds without closed nonspacelike geodesics, Proc. Amer. Math. Soc. 98 (1986), 119-123. MR0848888 (87i:53094)

[8] M. Guediri, J. Lafontaine, Sur la complétude des variétés pseudo-riemanniennes, J. Geom. Phys. 15 (1995), 150-158. MR.1310948 (96b:53083)

[9] M. Guediri, On the existence of closed timelike geodesics in compact spacetimes, Math. Z. 239 (2002), 277-291. MR 1888225 (2002k:53138)

[10] M. Guediri, On the existence of closed timelike geodesics in compact spacetimes. II, Math. Z. 244 (2003), 577-585. MR1992025 (2004d:53041)

[11] M. Guediri, On the nonexistence of closed timelike geodesics in flat Lorentz 2-step nilmanifolds, Trans. Amer. Math. Soc. 355 (2003), 775-786. MR.1932725(2003f:53058)

[12] M. Guediri, Lorentz geometry of 2-step nilpotent Lie groups, Geom. Dedicata 100 (2003), 10-51. MR2011112 (2004m:53123)

[13] S.W. Hawking, G.F.R. Ellis, The large scale structure of spacetime, Cambridge Univ. Press, Cambridge, 1973. MR0424186 (54:12154) 
[14] W. Klingenberg, Riemannian geometry, De Gruyter Studies in Mathematics, Berlin, 1982. MR0666697 (84j:53001)

[15] B. O'Neill, Semi-Riemannian geometry with applications to relativity, Academic Press, New York, 1983. MR0719023 (85f:53002)

[16] G. Oshikiri, Totally geodesic foliations and Killing fields, Tôhoku Math. J. 35 (1983), 387392. MR.0711354 (84j:53044)

[17] M. Sanchez, Structure of Lorentzian tori with a Killing vector field, Trans. Amer. Math. Soc. 349 (1997), 1063-1080. MR.1376554 (97f:53108)

[18] M. Spivak, A comprehensive introduction to differential geometry, Vol. IV, Publish or Perish, Boston, 1975. MR0394452 (52:15254a)

[19] F.T. Tipler, Existence of closed timelike geodesics in Lorentz spaces, Proc. Amer. Math. Soc. 76 (1979), 145-147. MR0534406 (80f:83016)

[20] B. Wegner, Zeitartige Geodätische Schleifen in Kompakten Lorentz-Mannigfaltigkeiten, Mathematical papers given on the occasion of Ernst Mohr's 75th birthday, Tech. Univ. Berlin (1985), 297-306. MR0809011 (87a:53100)

[21] J.A. Wolf, Spaces of constant curvature, Publish or Perish, Boston, 1974. MR0343214 $(49: 7958)$

Department of Mathematics, College of Science, King Saud University, P.O. Box 2455, Riyadh 11451, Saudi Arabia

E-mail address: mguediri@ksu.edu.sa 